\title{
Association between SNPs and gene expression in multiple regions of the human brain
}

\author{
S Kim ${ }^{1}, \mathrm{H} \mathrm{Cho}^{2}$, D Lee ${ }^{2}$ and MJ Webster ${ }^{1}$
}

Identifying the genetic cis associations between DNA variants (single-nucleotide polymorphisms (SNPs)) and gene expression in brain tissue may be a promising approach to find functionally relevant pathways that contribute to the etiology of psychiatric disorders. In this study, we examined the association between genetic variations and gene expression in prefrontal cortex, hippocampus, temporal cortex, thalamus and cerebellum in subjects with psychiatric disorders and in normal controls. We identified cis associations between 648 transcripts and 6725 SNPs in the various brain regions. Several SNPs showed brain regional-specific associations. The expression level of only one gene, PDE4DIP, was associated with a SNP, rs12124527, in all the brain regions tested here. From our data, we generated a list of brain cis expression quantitative trait loci (eQTL) genes that we compared with a list of schizophrenia candidate genes downloaded from the Schizophrenia Forum (SZgene) database (http:// www.szgene.org/). Of the SZgene candidate genes, we found that the expression levels of four genes, HTR2A, PLXNA2, SRR and TCF4, were significantly associated with cis SNPs in at least one brain region tested. One gene, SRR, was also involved in a coexpression module that we found to be associated with disease status. In addition, a substantial number of cis eQTL genes were also involved in the module, suggesting eQTL analysis of brain tissue may identify more reliable susceptibility genes for schizophrenia than case-control genetic association analyses. In an attempt to facilitate the identification of genetic variations that may underlie the etiology of major psychiatric disorders, we have integrated the brain eQTL results into a public and online database, Stanley Neuropathology Consortium Integrative Database (SNCID; http://sncid.stanleyresearch.org).

Translational Psychiatry (2012) 2, e113; doi:10.1038/tp.2012.42; published online 8 May 2012

\section{Introduction}

Schizophrenia, bipolar disorder and severe depression are common and highly disabling brain diseases caused by an interaction of genetic and environmental factors. ${ }^{1,2}$ However, despite enormous efforts, the genetic variations that contribute to these diseases and their environmental risk factors remain elusive. Genome-wide association studies have frequently been employed to identify susceptibility genes and single-nucleotide polymorphisms (SNPs) that may be associated with these mental disorders. ${ }^{3-5} \mathrm{~A}$ number of candidate genes for the disorders have been reported. For instance, a web resource for schizophrenia, the Schizophrenia Forum (SZgene) database (http://www.szgene.org/), includes results from 1727 genetic association studies and reports 1008 candidate genes and 8788 polymorphisms in the update on 15 April $2011 .{ }^{6}$ Despite the numerous candidate genes reported for schizophrenia, the effect size of each variant is small or moderate and most associated SNPs have failed to be replicated. The need for independent and systematic validation to prioritize further examination of possible candidate genes for mental disease is widely acknowledged.

Identification of DNA sequence variants that regulate gene expression levels in a relevant tissue is one of the most promising approaches used to initially scan for candidate genes as well as to prioritize previously identified candidate genes that are associated with complex disease such as psychiatric disorders. ${ }^{7-9}$ The identification of a cis association of a SNP with gene expression levels has been previously used to validate candidate genes for complex traits mapped to the same chromosomal locations. ${ }^{10}$ Our recent study using an integrative approach that combined results from genomewide SNP scans for the cytoarchitectural traits and cis expression quantitative trait loci (eQTL) analysis in the brain tissue revealed two novel candidate genes associated with cellular abnormalities in the prefrontal cortex of major psychiatric disorders. ${ }^{11}$ Limited availability of human postmortem brain tissues is a major obstacle to obtaining detailed brain expression complex trait loci (eQTL) mapping. Utilization of publicly available resources is an effective alternative strategy that may overcome such limitation. The Stanley Neuropathology Consortium Integrative Database (SNCID; http://sncid.stanleyresearch.org) is a publicly available and web-based tool that integrates expression microarray data sets from five brain regions including frontal cortex, temporal cortex, thalamus, cerebellum and hippocampus and genomewide SNP genotype data sets of subjects in the Stanley Neuropathology Consortium (SNC) and the Array Collection (AC). ${ }^{12} \mathrm{~A}$ total of 1749 neuropathology data sets using the

\footnotetext{
${ }^{1}$ Stanley Brain Research Laboratory, Stanley Medical Research Institute, Rockville, MD, USA and ${ }^{2}$ Department of Bio and Brain Engineering, KAIST, Yuseong-gu, Daejeon, Republic of Korea

Correspondence: Dr MJ Webster, Stanley Medical Research Institute, 9800 Medical Center Drive, Rockville, MD 20850, USA or Dr D Lee, KAIST, 291 Daehak-ro, Yuseong-gu, Daejeon 305-701, Republic of Korea.

E-mail: websterm@stanleyresearch.org or dhlee@ kaist.ac.kr

Keywords: cis SNP; eQTL; post-mortem brain; psychiatric disorders; schizophrenia; SNCID

Received 12 March 2012; accepted 10 April 2012
} 
SNC are integrated into the database, which thereby enables one to further explore the correlations between gene expression levels and quantitative measures of neuropathological markers in the various brain regions. The specific aims of this study are twofold. First, we explore the candidate genes that may be functionally relevant for major psychiatric disorders by identifying cis associations between SNPs and gene expression in various brain tissues. Second, we examine the possible functional role of schizophrenia candidate genes that were previously identified in genetic association studies. Thus, we explored cis eQTLs in the four brain regions, frontal cortex, temporal cortex, thalamus and cerebellum, of SNC subjects and in hippocampus of AC subjects. We also repeated the analysis in frontal cortex data from the $A C$ as a replication study to examine the overall consensus of cis eQTLs between the two frontal data sets. We then examined whether the expression levels of any candidate genes from the SZgene database meta-analysis (http://www.szgene.org/) were regulated by cis expressed SNPs (eSNPs) in brain tissues, in order to determine if there were any functional effects on gene expression of the previously identified schizophrenia susceptibility genes. Finally, we performed a coexpression network analysis between the genes in the frontal cortex that were differentially expressed between schizophrenia and normal controls and the cis eQTL genes in an attempt to identify the potential role of these genes in a disease-specific coexpression module.

\section{Materials and methods}

Data used in this study. Gene expression microarray data from frontal cortex, ${ }^{13}$ cerebellum, thalamus and temporal cortex ${ }^{14}$ were generated by multiple independent groups using samples from the SNC $(N=60)$, which contains 15 well-matched cases in each of four groups: schizophrenia, bipolar disorder, major depression and unaffected controls. ${ }^{15}$ Other sets of microarray data from frontal cortex ${ }^{16,17}$ and hippocampus were generated using samples from the $A C$ $(N=105)$. The $A C$ is an independent tissue collection containing 35 cases in each of three groups: schizophrenia, bipolar disorder and unaffected controls. The groups from both tissue collections are matched for descriptive variables such as age, gender, race, post-mortem interval, mRNA quality, brain $\mathrm{pH}$ and hemisphere. Outlier chip data were excluded in this analysis based on previous quality-control analyses for chip-level parameters such as scaling factor, gene call and average correlation. ${ }^{18}$ Information for the microarray studies such as tissue collection, brain region and number of outlier chips is listed in the Supplementary Table S1 online. The confounding effects on the Frozen Robust Multiarray Analysis (fRMA)-normalized microarray gene expression data were identified using Surrogate Variable Analysis (SVA). ${ }^{19}$ To adjust disease effect on the gene expression data, we randomly assign 0 or 1 for the primary variable in the SVA. All covariates from SVA were used in the linear regression to adjust the confounding effects on the gene expression data. The standardized residuals from the linear regression were used to evaluate the effectiveness of this method on removing confounding variables on two microarray data sets from both the SNC and AC. Transcripts correlated with potential confounding variables were identified using nonparametric analysis. The continuous variables such as age, brain $\mathrm{pH}$, post-mortem interval and lifetime exposure to antipsychotics were examined by correlation analysis using $R$ (open source program from Comprehensive R Archive Network (CRAN)). Two categorical variables such as microarray batch and sex were tested using variance analysis. Adjusted $P$-values, based on the Hochberg method that were $<0.05$, were considered significant. Although all cases and controls were included in the analysis, only the disorder cases were used for the correlation analysis for the effect of lifetime exposure to antipsychotics. SNP genotyping data using DNA samples from the SNC and the AC were generated by Dr Chun-Yu Liu and colleagues (University of Chicago, IL, USA) using the Human SNP Array 5.0 chips (Affymetrix, Santa Clara, CA, USA). ${ }^{20}$

eQTL analysis. Raw image files from SNP chips, qualitycontrol analysis and identification of ethnic outliers were performed as previously described. ${ }^{11}$ Briefly, genotypes were called using the BRLMM algorithm (Affymetrix). SNPS with a call rate of $<90 \%$, minor allele frequency $<5 \%$ or extreme deviation from Hardy-Weinberg equilibrium test $(P<0.05)$ were filtered out for further eQTL analyses. A total of 309531 SNPs passed this filter. For examination of population stratification, clustering was initially performed using the pairwise identity-by-state (IBS) calculator in the PLINK. ${ }^{21}$ IBS pairwise distances were then plotted and examined by multidimensional scaling analysis and $Z$ statistical analysis. Samples of $>3$ s.d. compared with the group mean were considered outliers. Four ethnic outliers from the SNC and three outliers from the AC were excluded in the eQTL analysis. One additional sample from AC was excluded because of a final diagnosis of CADASIL (cerebral autosomal dominant arteriopathy with subcortical infarcts and leukoencephalopathy). We only used genotyped SNP data from chips for our association analysis rather than imputing genotypes because SNP imputation can often result in errors in genotyping and cause false-positive associations. $^{22}$ The standardized residuals from the linear regression were used as traits in PLINK for eQTL analyses. We defined cis eSNPs as those that were localized within $1 \mathrm{Mb}$ of either the $5^{\prime}$ or the $3^{\prime}$ end of the gene. The trans eSNPs were defined as all SNPs that reached genome-wide significance level, except those in a cis position. We employed a conservative Bonferroni method to correct multiple testing for controlling false positives. ${ }^{7}$ Adjusted $P$-values of $<0.05$ (unadjusted $P$-value; $1.6 \mathrm{E}-07=0.05 / 309531$ ) were considered genome-wide significant for eQTL analyses.

Coexpression network analysis. Unsupervised and supervised coexpression network analyses were performed using the Weighted Correlation Network Analysis (WGCNA) in $R^{23}$ The coexpression network was generated using expression values of all genes in the frontal cortex of schizophrenia and normal controls from the AC (unsupervised WGCNA). A second coexpression network was generated using significant cis eQTL genes and genes that were differentially expressed in the frontal cortex between 
schizophrenia and normal controls from the AC samples (supervised WGCNA). ${ }^{24}$ A total of four microarray data sets (at www.stanleygenomics.org; study no. 1, 3, 5 and 7) were generated from prefrontal cortex. Three of these (study no. 1 , 3 and 7$)^{16,17}$ were generated using the same platform, Affymetrix $133 \mathrm{a}$, and hence to avoid variations between platforms we pooled the data from these three data sets. The pooled data were then subjected to median normalization with the biometric research branch (BRB)-array tools (http:// linus.nci.nih.gov/BRB-ArrayTools.html) to remove systematic variations. After median normalization, confounding effects were adjusted using SVA and a linear regression method as described in the previous section. However, disease effect was not removed. Standardized residuals that were significantly associated with disease (nominal $P$-value $<0.05$ ) and standardized residuals of cis eQTL genes were then used as input for the WGCNA. ${ }^{23}$ The minimum module size and the minimum height for merging modules were set at 30 and 0.25 , respectively. The coexpression module was visualized using VisANT. ${ }^{25}$

Functional annotation. The cis eQTL genes and genes that were involved in the coexpression module were functionally annotated using the Database for Annotation, Visualization and Integrated Discovery (DAVID) database (http://david.abcc.ncifcrf.gov/home.jsp) and by the overrepresentational analysis method. ${ }^{26}$ The biological processes of Gene Ontology Consortium (http://www.geneontology.org) were used for functional annotations. The $P$-values of $<0.05$ were considered significant.

\section{Results}

eQTL analysis in various human post-mortem brain tissues. Gene expression microarray data derived from post-mortem brain tissue are often confounded by uncontrolled biological, clinical and technical variables. ${ }^{27}$ Batch effect is particularly problematic and has been shown to significantly affect gene expression levels in microarray data. $^{28,29}$ To remove the effect of batch and other confounding variables in our gene expression microarray data, we normalized the data using the newly developed method, fRMA, followed by the SVA. ${ }^{19,30}$ We evaluated how effective this method was at removing confounding variables using two microarray data sets from both the SNC and AC (Supplementary Table S1 online). Using the data set from SNC temporal cortex (study 18) we found that microarray batch was the most significant confounding variable in both the RMA and fRMA-normalized data sets, with 947 and 1031 transcripts significantly correlated with batch, respectively (Supplementary Table S2 online). Using the data set from AC frontal cortex (study 1) we found that microarray batch and brain $\mathrm{pH}$ were both major confounding variables (Supplementary Table S3 online). The SVA successfully adjusted the effects of the confounding variables on both microarray data sets (Supplementary Table S2 and S3 online).

Using the SVA we obtained the standardized residuals from the linear regression with covariates and conducted a genome-wide eQTL analysis of various brain tissues. We used the standardized residuals as traits. We initially analyzed gene expression microarray data from frontal cortex, temporal cortex, thalamus and cerebellum from the SNC (Supplementary Table S1 online). Expression levels of a total of 53, 11, 84 and 27 genes were correlated with cis SNPs in the frontal cortex, temporal cortex, thalamus and cerebellum at genomewide significance level, respectively (nominal $P<1.6 \mathrm{E}-07$; Figure $1 \mathrm{a}$ and Supplementary Table S4 online). Among the cis eQTL genes, expression levels of 16, 0, 20 and 5 genes were also significantly associated with trans SNPs in the frontal cortex, temporal cortex, thalamus and cerebellum, respectively (Supplementary Table S5 online). In addition, correlations between the expression levels of 31, 1, 69 and 15 genes and cis SNPs were unique in the frontal cortex, temporal cortex, thalamus and cerebellum, respectively (Figure 1a). The expression level of only one gene, phosphodiesterase $4 D$ interacting protein (PDE4DIP), was associated with a SNP, rs12124527, in all the brain regions tested here. We then
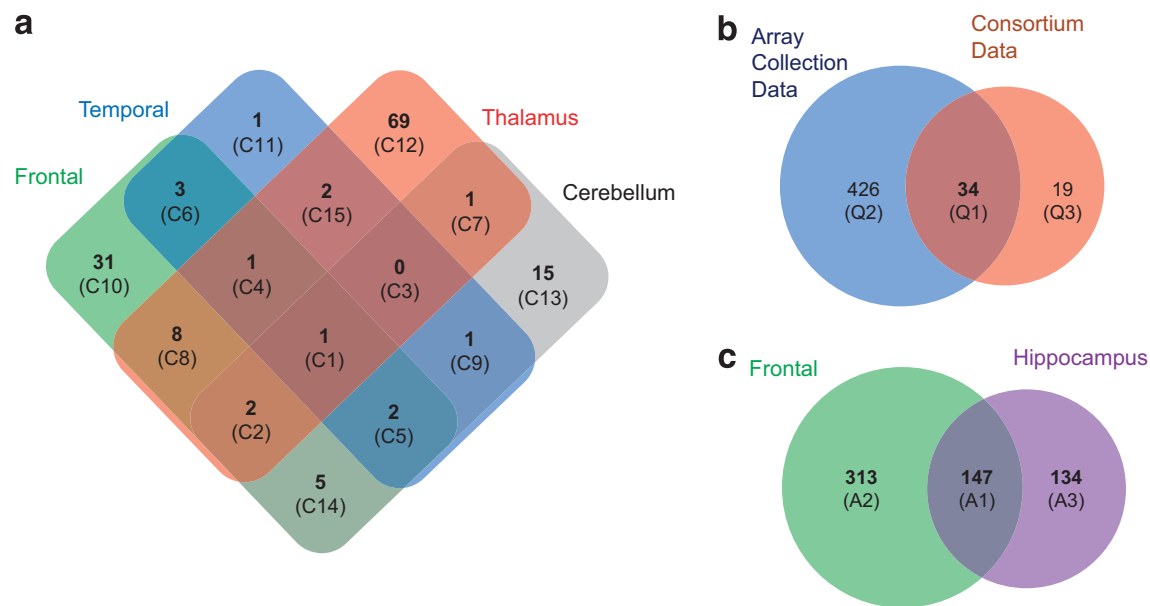

Figure 1 Number of cis expression quantitative trait loci (eQTL) genes in various brain regions. Venn diagram shows common and unique cis eQTL genes across multiple brain regions of the Stanley Neuropathology Consortium (SNC) samples (a) and of the Array Collection (AC) samples (c). Overlapped cis eQTL genes in the frontal cortex between the SNC samples and the AC samples are shown (b). 
Table 1 Biological processes (Gene ontology) significantly associated with cis eQTL genes in the frontal cortex of the Stanley Neuropathology Consortium samples

\begin{tabular}{lcc}
\hline Biological process categories & Count & Fraction (\%) \\
\hline GO:0007155-cell adhesion & 7 & 1.361868 \\
GO:0022610-biological adhesion & 7 & 1.361868 \\
GO:0007601-visual perception & 4 & 0.77821 \\
GO:0050953-sensory perception of light stimulus & 4 & 0.77821 \\
GO:0046907-intracellular transport & 6 & 1.167315 \\
GO:0035249-synaptic transmission, glutamatergic & 2 & 0.013 \\
\hline
\end{tabular}

Abbreviations: eQTL, expression quantitative trait loci; GO, Gene Ontology.

replicated the cis eQTLs of the frontal cortex using the larger AC collection. The replication study revealed associations between cis SNPs and expression levels of 460 genes and replicated 34 cis eQTL genes out of $53(64 \%)$ that were identified in the SNC study (Figure $1 \mathrm{~b}$ and Supplementary Table S6 online). Moreover, 281 cis eQTL genes were identified in the AC hippocampus data and 147 cis eQTLs were common to both the frontal cortex and hippocampus (Figure 1c and Supplementary Table S7 online). Among the cis eQTL genes, expression levels of 43 and 46 genes were also significantly associated with trans SNPs in the frontal cortex and hippocampus, respectively (Supplementary Table S5 online). The association between PDE4DIP expression and the rs12124527 SNP was replicated in the AC frontal cortex and hippocampal data.

Next, we performed a functional annotation analysis to identify biological processes that were overrepresented in the brain cis eQTL genes. Whereas several processes such as cell adhesion, visual perception and glutamatergic transmission were overrepresented in the genes with cis eSNPs in the SNC frontal cortex (Table 1), metabolic processes such as glutamine metabolic process and protein transport and targeting and antigen processing were overrepresented in the AC frontal cortex (Table 2). Amino acid metabolic process, nucleotide biosynthesis and enzyme-linked receptor protein signaling pathways were significantly overrepresented in cis eQTL genes in the AC hippocampus (Supplementary Table S8 online).

Comparison between schizophrenia susceptibility candidate genes and brain cis eQTL genes. Genetic association studies have yielded numerous candidate genes that may increase the risk for schizophrenia. However, most candidate genes have not been replicated nor functionally validated. To examine the possible functional role of schizophrenia candidate genes, we compared the list of the candidate genes in the SZgene database meta-analysis (updated 12/1/2010) to our list of cis eQTL genes. The SZgene meta-analysis identified 45 genetic variants and 42 linked genes. After excluding the non-SNP variants from their data set, we were left with 39 SNPs and 39 linked candidate genes. Because only 6 SNP markers out of the 39 SNPs were included in our Affymetrix SNP 5.0 data set, we conducted a gene-level comparison instead of SNP-level comparison. We determined whether there were cis associations between the expression levels of the 39 candidate genes and SNPs within $1 \mathrm{Mb}$ of the genes. Among the 39 candidate genes, we found that the expression levels of four genes, HTR2A, PLXNA2, SRR and TCF4, were significantly associated with cis SNPs in at least one brain region tested (Table 3). The expression levels of HR2A and PLXNA2 were associated with cis SNPs in the frontal cortex, whereas the expression levels of SRR (serine racemase) and TCF4 were associated with cis SNPs in two brain regions. The cis eSNPs of these genes are located at least $25 \mathrm{~kb}$ from the SNPs that were significantly associated with schizophrenia in the SZgene meta-analysis. Thus the SZgene case-control genetic association analyses for schizophrenia may not have identified the most functionally relevant genetic variations that contribute to the etiology of psychiatric disorders.

Coexpression network analysis in the frontal cortex. To further examine whether or not the four schizophrenia candidate genes (HTR2A, PLXNA2, SRR and TCF4) and genes of which expression levels were regulated by cis SNPs may be involved in the etiology of schizophrenia, we performed both unsupervised and supervised gene coexpression network analyses using the $A C$ frontal cortex data. We were unable to construct a coexpression module that was significantly associated with schizophrenia disease status using the unsupervised analysis. One module was associated with disease $(P=0.05)$; however, it was also associated with post-mortem interval $(P=0.01)$. We then constructed a supervised coexpression network using genes differentially expressed between schizophrenia and normal controls (Supplementary Table S9 online) and the cis eQTL genes obtained from the pooled data of three Affymetrix 133a microarray data sets that measured gene expression in the frontal cortex. We constructed one coexpression module that was significantly associated with schizophrenia disease status $(P=2 \mathrm{E}-08$; Figure 2a). Age, sex, post-mortem interval, brain $\mathrm{pH}$ and lifetime antipsychotic treatment were not significantly associated with this module (all $P>0.05$ ). Genes associated with apoptosis, chromatin organization, RNA splicing, cell cycle, regulation of nucleic acid metabolism and endocytosis were overrepresented in this module (Figure $2 \mathrm{~b}$ and Supplementary Table 10 online). A previous coexpression network analysis that used gene expression microarray data from prefrontal cortex from schizophrenia subjects and controls ${ }^{31}$ also identified a module (module 16) with similar overrepresentation of biological processes such as chromatin organization, cell cycle, endocytosis and regulation of nucleic acid metabolism. Apoptosis and endocytosis have previously been associated with the pathophysiology of the frontal cortex in schizophrenia, ${ }^{32-44}$ and recent studies have also indicated that aberrant RNA splicing and epigenetic alterations may be involved in the pathophysiology 
Table 2 Biological processes (Gene ontology) significantly associated with cis eQTL genes in the frontal cortex of the Array Collection samples

\begin{tabular}{|c|c|c|c|}
\hline Biological process categories & Count & Fraction (\%) & P-value \\
\hline GO:0006541-glutamine metabolic process & 6 & 0.13 & $5.00 \mathrm{E}-05$ \\
\hline GO:0009064-glutamine family amino acid metabolic process & 8 & 0.17 & $1.50 \mathrm{E}-04$ \\
\hline GO:0006412-translation & 19 & 0.41 & $5.10 \mathrm{E}-04$ \\
\hline GO:0046907-intracellular transport & 28 & 0.60 & $1.80 \mathrm{E}-03$ \\
\hline GO:0006414-translational elongation & 9 & 0.19 & $2.00 \mathrm{E}-03$ \\
\hline GO:0009069-serine family amino acid metabolic process & 5 & 0.11 & $2.50 \mathrm{E}-03$ \\
\hline GO:0034613-cellular protein localization & 19 & 0.41 & $5.50 \mathrm{E}-03$ \\
\hline GO:0070727-cellular macromolecule localization & 19 & 0.41 & $5.80 \mathrm{E}-03$ \\
\hline GO:0002474-antigen processing and presentation of peptide antigen via MHC class I & 4 & 0.09 & $6.00 \mathrm{E}-03$ \\
\hline GO:0002483-antigen processing and presentation of endogenous peptide antigen & 3 & 0.06 & $7.10 \mathrm{E}-03$ \\
\hline GO:0019885-antigen processing and presentation of endogenous peptide antigen via MHC class I & 3 & 0.06 & $7.10 \mathrm{E}-03$ \\
\hline GO:0006508-proteolysis & 37 & 0.79 & $7.60 \mathrm{E}-03$ \\
\hline GO:0006605-protein targeting & 12 & 0.26 & $9.50 \mathrm{E}-03$ \\
\hline GO:0006886-intracellular protein transport & 17 & 0.36 & $1.10 \mathrm{E}-02$ \\
\hline GO:0019882-antigen processing and presentation & 7 & 0.15 & $1.10 \mathrm{E}-02$ \\
\hline GO:0046777-protein amino acid autophosphorylation & 7 & 0.15 & $1.20 \mathrm{E}-02$ \\
\hline GO:0015031-protein transport & 28 & 0.60 & $1.30 \mathrm{E}-02$ \\
\hline GO:0019883-antigen processing and presentation of endogenous antigen & 3 & 0.06 & $1.30 \mathrm{E}-02$ \\
\hline GO:0045184-establishment of protein localization & 28 & 0.60 & $1.40 \mathrm{E}-02$ \\
\hline GO:0051603-proteolysis involved in cellular protein catabolic process & 23 & 0.49 & $1.70 \mathrm{E}-02$ \\
\hline GO:0044257-cellular protein catabolic process & 23 & 0.49 & $1.70 \mathrm{E}-02$ \\
\hline GO:0043632-modification-dependent macromolecule catabolic process & 22 & 0.47 & $2.00 \mathrm{E}-02$ \\
\hline GO:0019941-modification-dependent protein catabolic process & 22 & 0.47 & $2.00 \mathrm{E}-02$ \\
\hline GO:0001510-RNA methylation & 3 & 0.06 & $2.00 \mathrm{E}-02$ \\
\hline GO:0044265-cellular macromolecule catabolic process & 26 & 0.56 & $2.30 \mathrm{E}-02$ \\
\hline GO:0030163-protein catabolic process & 23 & 0.49 & $2.40 \mathrm{E}-02$ \\
\hline GO:0009070-serine family amino acid biosynthetic process & 3 & 0.06 & $2.40 \mathrm{E}-02$ \\
\hline GO:0048002-antigen processing and presentation of peptide antigen & 4 & 0.09 & $2.40 \mathrm{E}-02$ \\
\hline GO:0008104-protein localization & 30 & 0.64 & $2.50 \mathrm{E}-02$ \\
\hline GO:0007143-female meiosis & 3 & 0.06 & $2.90 \mathrm{E}-02$ \\
\hline GO:0044271-nitrogen compound biosynthetic process & 14 & 0.30 & $3.20 \mathrm{E}-02$ \\
\hline GO:0006607-NLS-bearing substrate import into nucleus & 3 & 0.06 & $3.30 \mathrm{E}-02$ \\
\hline GO:0034660-ncRNA metabolic process & 11 & 0.24 & $3.50 \mathrm{E}-02$ \\
\hline GO:0006625-protein targeting to peroxisome & 3 & 0.06 & $3.80 \mathrm{E}-02$ \\
\hline GO:0043574-peroxisomal transport & 3 & 0.06 & $4.40 \mathrm{E}-02$ \\
\hline GO:0009057-macromolecule catabolic process & 26 & 0.56 & $4.70 \mathrm{E}-02$ \\
\hline GO:0006399-tRNA metabolic process & 7 & 0.15 & $5.00 \mathrm{E}-02$ \\
\hline
\end{tabular}

Abbreviations: eQTL, expression quantitative trait loci; GO, Gene Ontology; MHC, major histocompatibility complex; NLS, nuclear localization signal; tRNA, transfer RNA.

Table 3 Schizophrenia candidate genes (from the SZgene database) with expression levels significantly associated with cis eSNPs in brain tissue

\begin{tabular}{|c|c|c|c|c|c|c|c|c|}
\hline Symbol & Chr. & Position $^{\mathrm{a}}$ & Study_ID & Brain_Region & SNP & S_Position & P-value & Adjusted P-value \\
\hline HTR2A & 13 & (R): $47407513-47470175$ & Study01 & Frontal cortex & rs1923882 & 47411661 & $3.08 \mathrm{E}-10$ & $9.45 \mathrm{E}-05$ \\
\hline & 13 & $407513-47470175$ & Study07 & Frontal cortex & rs1923882 & 47411661 & -08 & 0 \\
\hline PLXNA2 & 1 & (R): $208195587-208417665$ & Study01 & Frontal cortex & rs6659522 & 208199100 & $3.26 \mathrm{E}-10$ & $1.00 \mathrm{E}-04$ \\
\hline PLXNA2 & 1 & (R): $208195587-208417665$ & Study03 & Frontal cortex & rs6659522 & 208199100 & $1.03 \mathrm{E}-09$ & $3.15 \mathrm{E}-04$ \\
\hline$P L X N A 2$ & 1 & (R): $208195587-208417665$ & Study07 & Frontal cortex & rs6702082 & 208206946 & $4.04 \mathrm{E}-08$ & $1.24 \mathrm{E}-02$ \\
\hline SRR & 17 & $(F): 2207248-2228553$ & Study01 & Frontal cortex & rs16952025 & 2116798 & $3.24 \mathrm{E}-08$ & $9.94 \mathrm{E}-03$ \\
\hline$S R R$ & 17 & $(F): 2207248-2228553$ & Study03 & Frontal cortex & rs16952025 & 2116798 & $8.86 \mathrm{E}-08$ & $2.72 \mathrm{E}-02$ \\
\hline$S R R$ & 17 & $\left(F^{\prime}\right): 2207248-2228553$ & Study07 & Frontal cortex & rs16952025 & 2116798 & $1.65 \mathrm{E}-13$ & $5.06 \mathrm{E}-08$ \\
\hline SRR & 17 & $(F): 2207248-2228553$ & Study 17 & Hippocampus & rs16952025 & 2116798 & $9.60 \mathrm{E}-11$ & $2.95 \mathrm{E}-05$ \\
\hline TCF4 & 18 & (R): $52889562-53255860$ & Study01 & Frontal cortex & rs1261085 & 52889967 & $1.08 \mathrm{E}-08$ & $3.33 \mathrm{E}-03$ \\
\hline TCF4 & 18 & (R): $52889562-53255860$ & Study03 & Frontal cortex & rs1261134 & 52931763 & $1.98 \mathrm{E}-15$ & $6.09 \mathrm{E}-10$ \\
\hline TCF4 & 18 & (R): $52889562-53255860$ & Study05 & Frontal cortex & rs1261073 & 52907820 & $2.18 \mathrm{E}-12$ & $6.68 \mathrm{E}-07$ \\
\hline TCF4 & 18 & (R): $52889562-53255860$ & Study07 & Frontal cortex & rs1261073 & 52907820 & $1.57 \mathrm{E}-15$ & $4.82 \mathrm{E}-10$ \\
\hline TCF4 & 18 & (R): $52889562-53255860$ & Study 16 & Thalamus & rs1261134 & 52931763 & $3.86 \mathrm{E}-08$ & $1.18 \mathrm{E}-02$ \\
\hline
\end{tabular}

Abbreviation: eSNP, expressed single-nucleotide polymorphism.

Genome build;hg19. ${ }^{\mathrm{F}} \mathrm{F}$ and $\mathrm{R}$ represent the forward orientation or reverse orientation on a chromosome. ${ }^{\mathrm{b}}$ Adjusted $P$-value using Bonferroni method.

of schizophrenia. ${ }^{35,36}$ Several genes associated with GABAergic neurons, including $\gamma$-aminobutyric acid (GABA) A receptor, $\delta(G A B R D)$ and parvalbumin $(P V A L B)$, were found in the coexpression module. However, out of the four schizophrenia candidate genes common to both the SZgene meta-analysis and our cis eQTL gene list, only one, SRR, was found in the module. The biological process, response to drug, was overrepresented in the module and was enriched with 11 genes including SRR. A substantial number of cis eQTL genes were involved in the coexpression module 



Figure 2 Coexpression network analysis in the frontal cortex. The coexpression module that is significantly associated with schizophrenia in frontal cortex of the Array Collection (AC) (a) and biological processes (Gene ontology) overrepresented in the genes in the coexpression module (b). Network connections with topological overlap above the threshold of 0.02 were visualized using VisANT.$^{25}$ The cis expression quantitative trait loci (eQTL) genes are pink. The candidate gene, SRR (serine racemase), derived from the meta-analyses of genetic studies in the SZgene database (http://www.szgene.org/) is in blue.

that was significantly associated with schizophrenia disease status and were also associated with the biological processes. This result indicates that cis eQTL analysis in brain tissue may more reliably identify susceptibility genes for schizophrenia as compared with the current case-control genetic association studies.

\section{Discussion}

Identifying genetic variations that affect gene expression in the brain may be a promising approach for finding molecular pathways that are functionally relevant to the etiology and/or treatment of mental disease. In this study, we conducted an eQTL analysis of 315440 transcripts in 5 different brain regions from two different tissue collections and identified cis associations between 648 transcripts and 6725 SNPs. The expression of one gene, PDE4DIP, was associated with one SNP, rs12124527, in all brain regions examined. This association was also previously described in the frontal cortex. ${ }^{20}$ The protein encoded by PDE4DIP serves to anchor phosphodiesterase $4 \mathrm{D}$ to the Golgi/centrosome region of the cell. A number of abnormalities in the phosphodiesterase signaling system have been described in the brains of subjects with schizophrenia, bipolar disorder and depression, ${ }^{37-42}$ indicating that molecules within this system could be potential targets for therapeutic intervention. ${ }^{38,43}$

Approximately $14 \%$ of cis eQTL genes were also correlated with trans SNPs in various brain regions, suggesting that the expression levels of a subset of cis eQTL genes may be regulated by multiple variants. However, when we examined whether or not the expression levels of candidate genes from the SZgene database meta-analysis were significantly associated with cis SNPs, we found only 4 genes that overlapped between the SZgene database and our eQTL gene list. Furthermore, only one candidate gene, SRR, was involved in a coexpression module that was associated with schizophrenia. SRR maps to chromosome $17 \mathrm{p} 13$ and encodes an enzyme that synthesizes D-serine from L-serine. ${ }^{44}$ The $\mathrm{D}$-serine is an endogenous co-agonist of the $\mathrm{N}$-methyl-Daspartate (NMDA) receptor. ${ }^{45}$ Hypofunction of the NMDA receptor is potentially a major underlying pathophysiology of schizophrenia. ${ }^{46,47}$ Our results support this hypothesis and suggest that abnormal NMDA receptor-mediated signaling may be influenced by genetic variations. A SNP, rs16952025, localized in an intron of the neighboring gene, SMG6, was significantly associated with the expression level of SRR. However, there was no significant association between this SNP and the expression level of SMG6. Several post-mortem studies have examined levels of SRR mRNA and serine racemase protein in schizophrenia ${ }^{48}$ and found abnormalities in schizophrenia, although the results have been inconsistent. Although SRR mRNA levels appear to be unchanged in frontal cortex of schizophrenia, ${ }^{41}$ the protein levels have been reported to be either decreased, ${ }^{49}$ increased $^{50}$ or unchanged. ${ }^{51}$ The inconsistent results are most likely because of different methodologies, different cohorts (often with small numbers) and the different brain areas used. Consequently further study will be required in the future when larger cohorts become available to confirm changes in SRR levels in the brain of subjects with mental illness.

Our comprehensive brain eQTL analysis functionally validated only 4 genes out of 39 candidate genes positively identified in the SZgene meta-analysis. We were unable to identify any significant associations between the expression levels of the remaining genes and cis SNPs in any of the brain regions we tested. In fact, the 39 candidate genes were derived from 1008 candidate genes that were obtained from 1727 original genetic association studies. Such a low functional validation rate raises the possibility that the current case-control genetic association studies may not effectively 
identify genetic variations that underlie the etiology of schizophrenia. However, there are other reasons that may contribute to a low functional validation rate. For example, the probes on the microarray platforms used to analyze gene expression in this study mainly bind to sequences in the $3^{\prime}$-untranslated regions and do not distinguish between various alternative splicing isoforms. Indeed, tissue-specific alternative RNA-splicing is very predominant in the brain. ${ }^{52}$ Furthermore, intronic SNPs can be associated with the altered expression of specific alternative splicing isoforms of certain schizophrenia candidate genes, for example, ErbB4 and GRM3. ${ }^{53,54}$ Therefore, comprehensive expression profiling that includes various alternative splicing isoforms using deep mRNA-sequencing technology may aid in the identification of novel cis eQTL genes in human post-mortem brain tissues in the future.

The frontal cortex is one of the most thoroughly examined brain regions in post-mortem studies and many neuropathology abnormalities have been identified in this region in schizophrenia. ${ }^{55,56}$ Previous gene expression microarray studies in the frontal cortex identified several biological processes that were overrepresented in the genes differentially expressed between schizophrenia and normal controls; for example, decreased presynaptic function, abnormal mitochondrial function and altered expression of apoptosisrelated genes are all major findings from microarray studies of frontal cortex in schizophrenia. ${ }^{33,34,57}$ However, glutamatergic transmission, amino acid metabolism, proteolysis and protein targeting were all overrepresented in the eQTL genes in the frontal cortex in our current study. Thus, the abnormalities described in the biological pathways from the eQTL study may be more directly related to genetic variation, whereas the pathways identified by gene expression studies are likely to be influenced by factors in addition to genetic variation, including epigenetics and environmental factors.

Although our study reveals a number of associations between cis SNPs and gene expression in multiple brain regions, the results should be interpreted with caution. First, the SNC, which we used for the initial eQTL analyses, contains a relatively small sample size $(N=56)$. Small sample size is known to generate higher false-positive associations as well as to be a cause of low detection power in genome-wide association analysis. Thus, the cis eQTL results from frontal cortex, cerebellum, thalamus, and temporal cortex using SNC samples should be viewed as exploratory. However, we subsequently performed a second analysis using an independent collection $(A C)$ with a larger sample size $(N=101)$. Our previous power analysis using $A C$ as well as a previous eSNP association study indicated that a relatively small sample size $(N=100)$ has $>80 \%$ power to detect an association of gene expression traits with moderate effect size $\left(R^{2}=0.35\right) .{ }^{11,58}$ We therefore attempted to replicate the results of cis eQTLs in frontal cortex from SNC using the AC data. A total of $34(64 \%)$ of the cis eQTL genes identified in the SNC frontal cortex data were also found in the $A C$ frontal data. However, we identified 450 additional cis eQTL genes in the AC frontal cortex samples, which were not identified in the SNC frontal samples, suggesting that some significant cis eQTL genes may have been missed in the SNC analysis.
Second, using whole tissues for gene expression traits may dilute the effect of some genetic variants that may only act on cell type-specific gene expression. Although this phenomena has not been explored in the brain, there are numerous cell type-specific abnormalities in the brain of subjects with psychiatric disorders. ${ }^{32,59-61}$ Thus, the use of cell typespecific expression traits in future studies may increase the power to identify cis eQTLs in the brain.

In this study, we investigated the associations between SNPs and gene expression in various human brain regions. Although previous brain eQTL studies focused on cortex, ${ }^{8,62}$ we have extended the analysis to include the hippocampus, thalamus and cerebellum. These data can be used to identify genetic variations associated with psychiatric disorder and can be used to identify genetic variations that affect neuropathological abnormalities and gene expression changes. As we show in this study, the data can be used to functionally validate candidate genes to determine if they are affecting changes in gene expression in subjects with neuropsychiatric disorders. In order to facilitate further studies, we have integrated the genome-wide eQTL results from this study into the SNCID, which is a web-based database that also includes 1747 neuropathological markers measured in the same SNC samples. The update will allow users to investigate associations between SNPs and genes of interests in various brain regions and to further explore associations between SNPs and neuropathological markers and gene expression traits that are correlated with neuropathological markers in the various brain regions of subject with major psychiatric disorders.

\section{Conflict of interest}

The authors declare no conflict of interest.

Acknowledgements. We thank all the investigators who generated the original data in the SNCID, and their many collaborators, who made this database possible. We also thank all the technicians in the SMRI brain laboratory who prepared the brain tissues and extracted the RNA and DNA from the tissues. We specially thank the Keymind Company for their technical assistance with the database, in particular Marvin Suo. We thank Dr Horvath for helpful comments on network analysis using the WGCNA. HC and DL were supported by the World Class University program (R32-2008-000-10218-0) of the Ministry of Education, Science and Technology through the National Research Foundation of Korea.

1. Mueser KT, McGurk SR. Schizophrenia. Lancet 2004; 363: 2063-2072.

2. Sullivan PF, Neale MC, Kendler KS. Genetic epidemiology of major depression: review and meta-analysis. Am J Psychiatry 2000; 157: 1552-1562.

3. Purcell SM, Wray NR, Stone JL, Visscher PM, O'Donovan MC, Sullivan PF et al. Common polygenic variation contributes to risk of schizophrenia and bipolar disorder. Nature 2009; 460: 748-752.

4. Stefansson H, Ophoff RA, Steinberg S, Andreassen OA, Cichon S, Rujescu D et al. Common variants conferring risk of schizophrenia. Nature 2009; 460: 744-747.

5. Sklar P, Smoller JW, Fan J, Ferreira MA, Perlis RH, Chambert $\mathrm{K}$ et al. Whole-genome association study of bipolar disorder. Mol Psychiatry 2008; 13: 558-569.

6. Allen NC, Bagade S, McQueen MB, loannidis JP, Kavvoura FK, Khoury MJ et al. Systematic meta-analyses and field synopsis of genetic association studies in schizophrenia: the SzGene database. Nat Genet 2008; 40: 827-834.

7. Dixon AL, Liang L, Moffatt MF, Chen W, Heath S, Wong KC et al. A genome-wide association study of global gene expression. Nat Genet 2007; 39: 1202-1207.

8. Myers AJ, Gibbs JR, Webster JA, Rohrer K, Zhao A, Marlowe $L$ et al. A survey of genetic human cortical gene expression. Nat Genet 2007; 39: 1494-1499.

9. Stranger BE, Nica AC, Forrest MS, Dimas A, Bird CP, Beazley C et al. Population genomics of human gene expression. Nat Genet 2007; 39: 1217-1224.

10. Emilsson V, Thorleifsson G, Zhang B, Leonardson AS, Zink F, Zhu J et al. Genetics of gene expression and its effect on disease. Nature 2008; 452: 423-428. 
11. Kim S, Webster MJ. Integrative genome-wide association analysis of cytoarchitectural abnormalities in the prefrontal cortex of psychiatric disorders. Mol Psychiatry 2010; 16: 452-461.

12. Kim S, Webster MJ. The stanley neuropathology consortium integrative database: a novel, web-based tool for exploring neuropathological markers in psychiatric disorders and the biological processes associated with abnormalities of those markers. Neuropsychopharmacology 2010; 35: 473-482.

13. Tkachev D, Mimmack ML, Ryan MM, Wayland M, Freeman T, Jones PB et al. Oligodendrocyte dysfunction in schizophrenia and bipolar disorder. Lancet 2003; 362: 798-805.

14. Aston C, Jiang L, Sokolov BP. Microarray analysis of postmortem temporal cortex from patients with schizophrenia. J Neurosci Res 2004; 77: 858-866.

15. Torrey EF, Webster M, Knable M, Johnston N, Yolken RH. The stanley foundation brain collection and neuropathology consortium. Schizophr Res 2000; 44: 151-155.

16. Iwamoto $\mathrm{K}$, Bundo $\mathrm{M}$, Kato $\mathrm{T}$. Altered expression of mitochondria-related genes in postmortem brains of patients with bipolar disorder or schizophrenia, as revealed by largescale DNA microarray analysis. Hum Mol Genet 2005; 14: 241-253.

17. Ryan MM, Lockstone HE, Huffaker SJ, Wayland MT, Webster MJ, Bahn S. Gene expression analysis of bipolar disorder reveals downregulation of the ubiquitin cycle and alterations in synaptic genes. Mol Psychiatry 2006; 11: 965-978.

18. Higgs BW, Elashoff M, Richman S, Barci B. An online database for brain disease research. BMC Genomics 2006; 7: 70.

19. Leek JT, Storey JD. Capturing heterogeneity in gene expression studies by surrogate variable analysis. PLOS Genet 2007; 3: 1724-1735.

20. Liu C, Cheng L, Badner JA, Zhang D, Craig DW, Redman M et al. Whole-genome association mapping of gene expression in the human prefrontal cortex. Mol Psychiatry 2010; 15: 779-784.

21. Purcell S, Neale B, Todd-Brown K, Thomas L, Ferreira MA, Bender D et al. PLINK: a tool set for whole-genome association and population-based linkage analyses. Am J Hum Genet 2007; 81: 559-575.

22. Pei YF, Li J, Zhang L, Papasian CJ, Deng HW. Analyses and comparison of accuracy of different genotype imputation methods. PLOS One 2008; 3: e3551.

23. Langfelder $P$, Horvath $S$. WGCNA: an $R$ package for weighted correlation network analysis. BMC Bioinformatics 2008; 9: 559

24. Gargalovic PS, Imura M, Zhang B, Gharavi NM, Clark MJ, Pagnon J et al. Identification of inflammatory gene modules based on variations of human endothelial cell responses to oxidized lipids. Proc Natl Acad Sci USA 2006; 103: 12741-12746.

25. Hu Z, Mellor J, Wu J, DeLisi C. VisANT: an online visualization and analysis tool for biological interaction data. BMC Bioinformatics 2004; 5: 17.

26. Dennis Jr G, Sherman BT, Hosack DA, Yang J, Gao W, Lane HC et al. DAVID: Database for Annotation, Visualization, and Integrated Discovery. Genome Biol 2003; 4: P3.

27. Mirnics K, Levitt $P$, Lewis DA. DNA microarray analysis of postmortem brain tissue. Int Rev Neurobiol 2004; 60: 153-181.

28. Fare TL, Coffey EM, Dai H, He YD, Kessler DA, Kilian KA et al. Effects of atmospheric ozone on microarray data quality. Anal Chem 2003; 75: 4672-4675.

29. Leek JT, Scharpf RB, Bravo HC, Simcha D, Langmead B, Johnson WE et al. Tackling the widespread and critical impact of batch effects in high-throughput data. Nat Rev Genet 2010; 11: 733-739.

30. McCall MN, Bolstad BM, Irizarry RA. Frozen robust multiarray analysis (fRMA). Biostatistics 2010; 11: 242-253.

31. Torkamani A, Dean B, Schork NJ, Thomas EA. Coexpression network analysis of neural tissue reveals perturbations in developmental processes in schizophrenia. Genome Res 2010; 20: 403-412

32. Uranova NA, Vostrikov VM, Orlovskaya DD, Rachmanova VI. Oligodendroglial density in the prefrontal cortex in schizophrenia and mood disorders: a study from the Stanley Neuropathology Consortium. Schizophr Res 2004; 67: 269-275.

33. Mirnics K, Middleton FA, Marquez A, Lewis DA, Levitt P. Molecular characterization of schizophrenia viewed by microarray analysis of gene expression in prefrontal cortex. Neuron 2000; 28: 53-67.

34. Kim S, Webster MJ. Correlation analysis between genome-wide expression profiles and cytoarchitectural abnormalities in the prefrontal cortex of psychiatric disorders. Mol Psychiatry 2010; 15: 326-336.

35. McInnes LA, Lauriat TL. RNA metabolism and dysmyelination in schizophrenia. Neurosci Biobehav Rev 2006; 30: 551-561.

36. Huang HS, Matevossian A, Whittle C, Kim SY, Schumacher A, Baker SP et al. Prefrontal dysfunction in schizophrenia involves mixed-lineage leukemia 1-regulated histone methylation at GABAergic gene promoters. J Neurosci 2007; 27: 11254-11262.

37. Millar JK, Pickard BS, Mackie S, James R, Christie S, Buchanan SR et al. DISC1 and $\mathrm{PDE} 4 \mathrm{~B}$ are interacting genetic factors in schizophrenia that regulate CAMP signaling Science 2005; 310: 1187-1191.

38. Wong ML, Whelan F, Deloukas P, Whittaker P, Delgado M, Cantor RM et al. Phosphodiesterase genes are associated with susceptibility to major depression and antidepressant treatment response. Proc Natl Acad Sci USA 2006; 103: 15124-15129.

39. Fatemi SH, King DP, Reutiman TJ, Folsom TD, Laurence JA, Lee $S$ et al. PDE4B polymorphisms and decreased PDE4B expression are associated with schizophrenia. Schizophr Res 2008; 101: 36-49.

40. Fatemi SH, Reutiman TJ, Folsom TD, Lee S. Phosphodiesterase-4A expression is reduced in cerebella of patients with bipolar disorder. Psychiatr Genet 2008; 18: 282-288.

41. Fatemi SH, Folsom TD, Reutiman TJ, Vazquez G. Phosphodiesterase signaling system is disrupted in the cerebella of subjects with schizophrenia, bipolar disorder, and major depression. Schizophr Res 2009; 119: 266-267.

42. Numata S, Iga J, Nakataki M, Tayoshi S, Taniguchi K, Sumitani S et al. Gene expression and association analyses of the phosphodiesterase 4B (PDE4B) gene in major depressive disorder in the Japanese population. Am J Med Genet B Neuropsychiatr Genet 2009; 150B: 527-534.

43. Hennah W, Porteous D. The DISC1 pathway modulates expression of neurodevelopmental, synaptogenic and sensory perception genes. PLoS One 2009; 4: e4906.

44. Wolosker H, Blackshaw S, Snyder SH. Serine racemase: a glial enzyme synthesizing Dserine to regulate glutamate-N-methyl-D-aspartate neurotransmission. Proc Natl Acad Sci USA 1999; 96: 13409-13414.

45. Leeson PD, Iversen LL. The glycine site on the NMDA receptor: structure-activity relationships and therapeutic potential. J Med Chem 1994; 37: 4053-4067.

46. Olney JW, Newcomer JW, Farber NB. NMDA receptor hypofunction model of schizophrenia. J Psychiatr Res 1999; 33: 523-533.

47. Belforte JE, Zsiros V, Sklar ER, Jiang Z, Yu G, Li Y et al. Postnatal NMDA receptor ablation in corticolimbic interneurons confers schizophrenia-like phenotypes. Nat Neurosci 2010; 13: $76-83$.

48. Verrall L, Burnet PW, Betts JF, Harrison PJ. The neurobiology of D-amino acid oxidase and its involvement in schizophrenia. Mol Psychiatry 2010; 15: 122-137.

49. Bendikov I, Nadri C, Amar S, Panizzutti R, De Miranda J, Wolosker H et al. A CSF and postmortem brain study of D-serine metabolic parameters in schizophrenia. Schizophr Res 2007; 90: 41-51.

50. Verrall L, Walker M, Rawlings N, Benzel I, Kew JN, Harrison PJ et al. d-Amino acid oxidase and serine racemase in human brain: normal distribution and altered expression in schizophrenia. Eur J Neurosci 2007; 26: 1657-1669.

51. Steffek AE, Haroutunian V, Meador-Woodruff JH. Serine racemase protein expression in cortex and hippocampus in schizophrenia. NeuroReport 2006; 17: 1181-1185.

52. Grabowski PJ, Black DL. Alternative RNA splicing in the nervous system. Prog Neurobiol 2001; 65: 289-308.

53. Sartorius LJ, Weinberger DR, Hyde TM, Harrison PJ, Kleinman JE, Lipska BK. Expression of a GRM3 splice variant is increased in the dorsolateral prefrontal cortex of individuals carrying a schizophrenia risk SNP. Neuropsychopharmacology 2008; 33: 2626-2634.

54. Law AJ, Kleinman JE, Weinberger DR, Weickert CS. Disease-associated intronic variants in the ErbB4 gene are related to altered ErbB4 splice-variant expression in the brain in schizophrenia. Hum Mol Genet 2007; 16: 129-141.

55. Knable MB, Barci BM, Bartko JJ, Webster MJ, Torrey EF. Molecular abnormalities in the major psychiatric illnesses: Classification and Regression Tree (CRT) analysis of postmortem prefrontal markers. Mol Psychiatry 2002; 7: 392-404.

56. Knable MB, Torrey EF, Webster MJ, Bartko JJ. Multivariate analysis of prefrontal cortical data from the Stanley Foundation Neuropathology Consortium. Brain Res Bull 2001; 55: 651-659.

57. Prabakaran S, Swatton JE, Ryan MM, Huffaker SJ, Huang JT, Griffin JL et al. Mitochondrial dysfunction in schizophrenia: evidence for compromised brain metabolism and oxidative stress. Mol Psychiatry 2004; 9: 684-697, 643.

58. Cheung VG, Spielman RS, Ewens KG, Weber TM, Morley M, Burdick JT. Mapping determinants of human gene expression by regional and genome-wide association. Nature 2005; 437: 1365-1369.

59. Beasley CL, Zhang ZJ, Patten I, Reynolds GP. Selective deficits in prefrontal cortical GABAergic neurons in schizophrenia defined by the presence of calcium-binding proteins. Biol Psychiatry 2002; 52: 708-715.

60. Lewis DA, Hashimoto T, Morris HM. Cell and receptor type-specific alterations in markers of GABA neurotransmission in the prefrontal cortex of subjects with schizophrenia. Neurotox Res 2008; 14: 237-248.

61. Vostrikov VM, Uranova NA, Orlovskaya DD. Deficit of perineuronal oligodendrocytes in the prefrontal cortex in schizophrenia and mood disorders. Schizophr Res 2007; 94: 273-280.

62. Webster JA, Gibbs JR, Clarke J, Ray M, Zhang W, Holmans P et al. Genetic control of human brain transcript expression in Alzheimer disease. Am J Hum Genet 2009; 84: 445-458.
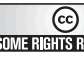

published by Nature Publishing Group. This work is licensed under the Creative Commons Attribution-Noncommercial-No Derivative Works 3.0 Unported License. To view a copy of this license, visit http://creativecommons.org/licenses/by-nc-nd/3.0/

\section{Supplementary Information accompanies the paper on the Translational Psychiatry website (http://www.nature.com/tp)}

